\title{
Left Ventricle Segmentation in Magnetic Resonance Images with Modified Active Contour Method
}

\author{
Maryam Aghai Amirkhizi ${ }^{1}$ \\ ${ }^{1}$ Department of Mechanical Engineering, Tabriz Branch, Islamic Azad University, Tabriz, Iran \\ maryam.amirkhizi@gmail.com \\ Siyamak Haghipour ${ }^{1}$ \\ ${ }^{1}$ Department of Mechanical Engineering, Tabriz Branch, Islamic Azad University, Tabriz, Iran \\ haghipour@iaut.ac.ir
}

\begin{abstract}
Desired segmentation of the image is a pivotal problem in image processing. Segmenting the left ventricle (LV) in magnetic resonance images (MRIs) is essential for evaluation of cardiac function. For the segmentation of cardiac MRI several methods have been proposed and implemented. Each of them has advantages and restrictions. A modified region-based active contour model was applied for segmentation of LV chamber. A new semi-automatic algorithm was suggested calculating the appropriate Balloon force according to mean intensity of the region of interest for each image. The database is included of 2,039 MR images collected from 18 children under 18 . The results were compared with previous literatures according to two standards: Dice Metric (DM) and Point to Curve (P2C). The obtained segmentation results are better than previously reported values in several literatures. In this study different points were used in cardiac cycle and several slice levels and classified into three levels: Base, Mid. and Apex. The best results were obtained at end diastole (ED) in comparis on with end systole (ES), and on base slice than other slices, because of LV bigger size in ED phase and base slice. With segmentation of LV MRI based on novel active contour and application of the suggested algorithm for balloon force calculation, the mean improvement of DM compared to Grosgeorge et al. is $19.6 \%$ in ED and $49.5 \%$ in ES phase. The mean improvement of $\mathrm{P} 2 \mathrm{C}$ compared with the same literature respectively for $\mathrm{ED}$ and $\mathrm{ES}$ phase is $43.8 \%$ and $39.6 \%$.
\end{abstract}

Index Terms - left ventricle Segmentation, MRI, Active contours, Dice metric, point to curve

\section{INTRODUCTION}

The Cardiovascular diseases are the main cause of death during the last decade in the world. Several techniques are available for heart disease diagnosis, such as: CT, Nuclear Medicine, Ultrasound and MRI. The capability of MRI is study possibility in only one examination the contraction and the viability through first-pass injection and delayed enhancement is its main superiority in comparison with other techniques. Because of its accuracy, MR has proven its advantage beyond nuclear methods particularly in the detection of sub endocardial death. Furthermore it is a noninvasive method with an excellent spatial separability of the contraction data, and an increasing time resolution according to the recent appearance of fast imaging sequences. [1]

The calculation of clinical parameters to estimate the cardiac function needs to segment the cardiac Ventricles. [2] There are two phases in each heartbeat: Systole or contraction phase and Diastole or expanding phase. In Systole phase, the blood reaches all organs except the heart and heart gets blood in diastole phase. In end Systole (ES) the heart has maximum shrinkage and at end diastole (ED) the heart has most expansion. So as the heart is a dynamic organ, the applied database is included of images in the whole cardiac cycle. The Database consists of Four-dimensional (4D) cardiac images -which incorporate knowledge about the 3D structure of the heart and its temporal deformation.

There are different causes for lower success of the algorithms described in the literatures for the segmentation and functional analysis of cardiac images in comparis on with what human experts done [4]:

1. The present methods do not combine enough amount of a basic knowledge regarding the segmentation problem.

2. Most of present methods do not bring up the threedimensional content as an integral part of their sufficiency.

3. Most of the present methods do not bring up the temporal content as an integral part of their functionality.

4. It is required the algorithm be enforceable for a wide range of image statuses. The variation in the images is because of patient health, patient motion and image noise and artifacts. [4]

Extensive study has been done about image segmentation and many techniques have been considered. [5-6] Between them, the Active contour 
method (ACM) [5, 7-10] is one of the most prospering methods. The basic idea of ACM is the evolution of a curve under some restrictions to capture the favorite object. According to the nature of restrictions, Zhang et al. , [11] classified the present ACMs into two types: edge-based models [5, 7-8, 10, 12-15] and region-based models. [9, 16-22]

A modified region-based active contour model (ACM) is applied in this paper. It is carried out with a special level set function called Selective Binary and Gaussian Filtering Regularized Level Set (SBGFRLS) method. Grosgeorge et al. [2] believe all cardiac MRI LV segmentation methods were encountered with following challenges:

(1) Fuzziness of the cavity borders because of blood flow, artifacts inception, and sectional volume effect chiefly for apical slices, (2) the attendance of papillary muscles in the LV pool. Due to the intricate crescent shape of the RV, which differs pursuant to the imaging slice level and because the RV function is less vital than the LV's, most research efforts have focused on the LV. [2] So we focus of LV segmentation too.

In the following subsection, we present an overview of active contours: region-based and edge-based. In Section 3, we present our experimental results for segmentation MRI LV. In Section 4, we conclude the paper.

\section{BACKGROUND}

The novel region-based active contour method applied in this paper is implemented with a new level set method called SBGFRL. The theoretical background of this method is explained as follows: GAC (Geodesic active contour) is one of the most common edge-based models [7-8], which applies gradient of an image to form an edge stopping function (ESF) to cease the contour evolvement on the object borders. Usually, a positive, subtractive and regular ESF $\mathrm{g}(|\nabla \mathrm{I}|)$ is applied

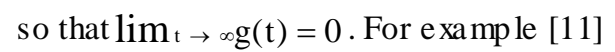

$$
\mathrm{g}(|\nabla \mathrm{I}|)=\frac{1}{1+|\nabla \mathrm{G} \sigma * \mathrm{I}|}
$$

Where $\mathrm{G} \sigma * \mathrm{I}$ identifies convolving between the image I and a Gaussian kernel with standard deviation $\sigma$. However, for digital images the discrete gradients are limited and then the ESF in (1) will never be zero on edges. So me edge-based ACMs presents a balloon force term to compress or stretch the contour, the design of the balloon force is difficult. The large balloon force will cause the contour pass via the weak edge of the object. On the other hand, too small balloon force maybe will bring the contour not pass via the narrow part of the object. [11]

A semi-automatic algorithm is suggested to calculate appropriate balloon force of each image. In this method, some training images are considered and assume multiplication of mean intensity into region of interest
(ROI) in the proper balloon force amount is a constant. Then user specifies ROI in other images and according to mean intensity of specified region and explained the relation between mean intensity and balloon force, the balloon force amount of each image will be calculated. By this method the time will be saved more than before.

Region-based active contour methods have more benefits in comparison with edge-based ones. The region-based models utilize the statistical information internal and external the contour which control the evolution, which have better efficiency for images with weak edges or no edges and have less sensitivity to noise. Also they are considerably less sensitive to the position of initial contour and can effectively detect the outside and inside borders simu ltaneously. [11]

One of the common region-based active contour models is C-V (Chan-Vese) model [9], which is based on the Mumford-Shah techniques for segmentation [8] and has been successfully applied to binary phase segmentation.

In this paper, a new region-based ACM is implemented, which consists of advantages of the $\mathrm{C}-\mathrm{V}$ and GAC models. This method uses the statistical information inside and outside the contour to make a region-based signed pressure force (SPF) function, [14] which can control the direction of initial contour evolution, to replace the ESF. The mentioned SPF function has opposite signs around the object borders, so when the contour is outside the object it can shrink or when inside the object, stretch.

A novel level set method named SBGFRLS is applied to implement this model. It modifies the usual level set methods by avoiding the computation of SDF and reinitialization. [23]

There is a step, which first penalizes level set function to be binary, and then utilizes a Gaussifitter for its regularization. With the Gausfilmar Level set function can be smoother and the evolution is more stable.

\section{A. The GAC and $C-V$ models}

\subsubsection{The GAC model}

Assume $\Omega$ be a bounded open subset of $\mathrm{R}^{2}$ and $\mathrm{I}:[0, \mathrm{a}] \times[0, \mathrm{~b}] \rightarrow \mathrm{R}^{+}$be a given image.

Assume $\mathrm{C}(\mathrm{q}):[0,1] \rightarrow \mathrm{R}^{2}$ be a parameterized planar curve in $\Omega$. The GAC model is formulated by minimizing the following energy functional: [11]

$$
\mathrm{E}^{\mathrm{GAC}}(\mathrm{C})=\int_{0}^{1} \mathrm{~g}(|\nabla \mathrm{I}(C(\mathrm{q}))|)\left|\mathrm{C}^{\prime}(\mathrm{q})\right| d \mathrm{q}
$$

Where $g$ is the ESF as in (1). Using the calculation of variation [24], we could get the Euler-Lagrange equation of (2) as follows:

$$
C_{t}=g(|\nabla \mathrm{I}|) \mathrm{k} \overrightarrow{\mathrm{N}}-(\nabla \mathrm{g} \cdot \overrightarrow{\mathrm{N}}) \overrightarrow{\mathrm{N}}
$$


Where $\mathrm{k}$ is the curvature of the contour and $\mathrm{N}$ is the inward normal to the curve. Usually a constant velocity term $\alpha$ is added to increase the extension velocity. So we can write (3) as follow:

$$
\mathrm{C}_{\mathrm{t}}=\mathrm{g}(|\nabla \mathrm{I}|)(\mathrm{k}+\alpha) \overrightarrow{\mathrm{N}}-(\nabla \mathrm{g} \cdot \overrightarrow{\mathrm{N}}) \overrightarrow{\mathrm{N}}
$$

The corresponding level set formulation is as follows:

$$
\frac{\partial \phi}{\partial \mathrm{t}} \equiv \mathrm{g}|\nabla \Phi|\left(\operatorname{div}\left(\frac{\nabla \Phi}{|\nabla \Phi|}\right)+\alpha\right)+\nabla g . \nabla \Phi
$$

Where $\alpha$ is the balloon force, which increases evolution speed and controls the contour shrinking or stretching.

\section{B. The $C-V$ model}

Chan and Vese [9] offered an active contour method which can be seen as a special case of the MumfordShah. [17] For image I in domain $\Omega$, the $\mathrm{C}-\mathrm{V}$ model obtained by minimizing the energy function as follow: [11]

$$
\mathrm{E}^{\mathrm{CV}}=\lambda_{1} \int_{\text {inside(c) }}\left|\mathrm{I}(\mathrm{x})-\mathrm{c}_{1}\right|^{2} \mathrm{dx}+\lambda_{2} \int_{\text {outside(c) }}\left|\mathrm{I}(\mathrm{x})-\mathrm{C}_{2}\right|^{2} \mathrm{dx}, \quad \mathrm{x} \in \Omega,
$$

Where $c_{1}$ and $c_{2}$ are constant and shows the average intensities inside and outside the contour, respectively. With the level set method, assume:

$$
\left\{\begin{array}{l}
\mathrm{C}=\{x \in \Omega: \Phi(x)=0\}, \\
\text { Inside }(C)=\{x \in \Omega: \Phi(x)>0\}, \\
\text { Outside }(C)=\{x \in \Omega: \Phi(x)<0\}
\end{array}\right.
$$

By minimizing Eq. (6), we solve $c_{1}$ and $c_{2}$ as follows:

$c_{1}(\Phi)=\frac{\int_{\Omega} \mathrm{I}(x) \cdot \mathrm{H}(\Phi) \mathrm{dx}}{\int_{\Omega} \mathrm{H}(\Phi) \mathrm{dx}}$

$\mathrm{c}_{2}(\Phi)=\frac{\int_{\Omega} \mathrm{I}(x) \cdot(1-\mathrm{H}(\Phi)) \mathrm{dx}}{\int_{\Omega}(1-H(\Phi)) \mathrm{dx}}$

By combining the length and area energy terms into (6) and minimizing them, we obtain the corresponding proportional level set formulation as follows:

$$
\frac{\partial \Phi}{\partial \mathrm{t}}=\delta(\Phi)\left[\mu \nabla\left(\frac{\nabla \Phi}{|\nabla \Phi|}\right)-v-\lambda_{1}\left(\mathrm{I}-\mathrm{c}_{1}\right)^{2}+\lambda_{2}\left(\mathrm{I}-\mathrm{c}_{2}\right)^{2}\right]
$$

Where $\mu \geq 0 \quad v \geq 0 \quad \lambda_{r_{1}}>0 \quad \lambda_{{ }^{2}}>0$ are fixed parameters, l controls the smoothness of zero level set, $\mathrm{m}$ increases the propagation speed, and $\lambda_{1}$ and $\lambda_{2}$ control the image data driven force inside and outside the contour, respectively. $\nabla$ is the gradient operator. $\mathrm{H}$ $(\Phi)$ is the Heaviside function and $\delta(\Phi)$ is the Dirac function. Generally, the regularized versions are selected as follows:

$$
\begin{aligned}
& \mathrm{H}_{\varepsilon}(\mathrm{Z})=\frac{1}{2}\left(1+\frac{2}{\pi} \arctan \left(\frac{\mathrm{z}}{\varepsilon}\right)\right), \\
& \delta_{\varepsilon}(\mathrm{Z})=\frac{1}{\pi} \cdot \frac{\varepsilon}{\varepsilon^{2}+\mathrm{z}^{2}}, \mathrm{Z} \in \mathrm{R}
\end{aligned}
$$

If $\varepsilon$ is too small, the values of $\delta_{\varepsilon}(Z)$ tend to be near zero to make its effective range small, so the energy functional has a tendency to fall into a local minimum. The object may fail to be extracted if the initial contour starts far from it. However, if e is large, although $\delta_{\varepsilon}(\mathrm{Z})$ tends to obtain a global minimum, the finial contour location may not be accurate.[25]

The SPF function defined in [14] has values in the range $[-1,1]$. It modulates the signs of the pressure forces inside and outside the region of interest so that the contour shrinks when outside the object, or expands when inside the object. Based on the analysis before, so the SPF function will be as follows:

$$
\operatorname{spf}(\mathrm{I}(\mathrm{x}))=\frac{\mathrm{I}(\mathrm{x})-\frac{\mathrm{c}_{1}+\mathrm{c}_{2}}{2}}{\max \left(\left|\mathrm{I}(\mathrm{x})-\frac{\mathrm{c}_{1}+\mathrm{c}_{2}}{2}\right|\right)}, \mathrm{x} \in \Omega,
$$

where $c_{1}$ and $c_{2}$ are defined in (7) and (8), respectively.

With replacing the SPF function in (11) with the ESF in (5), the level set formulation of the proposed model will be as follows:

$$
\begin{aligned}
& \frac{\partial \Phi}{\partial \mathrm{t}}=\operatorname{spf}(\mathrm{I}(\mathrm{x})) \cdot\left(\operatorname{div}\left(\frac{\nabla \Phi}{|\nabla \Phi|}\right)+\alpha\right)|\nabla \Phi|+\nabla \operatorname{spf}(\mathrm{I}(\mathrm{x})) \cdot \nabla \Phi, \\
& x \in \Omega
\end{aligned}
$$

Because we use a Gaussian filter to regularize the level set function to keep the interface smooth, the regular term $\operatorname{div}(\nabla \Phi /|\nabla \Phi|)|\nabla \Phi|$ is not required. Furthermore, as our model uses the statistical information of regions, the term $\nabla s p f . \nabla \phi$ in (12) can also be removed. Finally, the level set formulation of the proposed model can be written as follows:

$\frac{\partial \Phi}{\partial \mathrm{t}}=\operatorname{spf}(\mathrm{I}(\mathrm{x})) \cdot \alpha|\nabla \Phi|, x \in \Omega$

The main process for implementation of the proposed algorith $m$ is as follows:

1. Initialize the level set function as 


$$
\Phi(\mathrm{x}, \mathrm{t}=0)= \begin{cases}-\rho & x \in \Omega_{0}-\partial \Omega_{0}, \\ 0 & x \in \partial \Omega_{0}, \\ \rho & x \in \Omega-\Omega_{0},\end{cases}
$$

Where $\rho>0$ is a constant, $\Omega 0$ is a subset in the image domain $\Omega$ and $\partial \Omega 0$ is the boundary of $\Omega 0$

2. Compute $\mathrm{c} 1(\Phi)$ and $\mathrm{c} 2(\Phi)$ using (7) and (8), respectively.

3. Calculate appropriate $\alpha$ (balloon force) according to mean intensity of ROI

4. Evolve the level set function according to (13).

5. Let $\Phi=1$ if $\Phi>0$; otherwise, $\Phi=-1$.

6. Regularize the level set function with a Gaussian filter, i.e. $\Phi=\Phi^{*}$ Go (we assume standard deviation of Gaussian filter equal to 1.8)

7. Check whether the evolution of the level set function has converged. If not, return to step 2 .

\section{EXPERIMENTAL RESULTS}

The applied cardiac MRI database includes 18 subjects with 2039 images collected from children under the age of 18. Some of them have a normal cardiac function and some have abnormalities like aortic regurgitation, Enlarged ventricles, etc. The database was shared by Dr. Andreoupolos et al..[4] The images were scanned by a GE Genesis Signa MR scanner according to FIESTA scan protocol. Each patient's database consisted of exactly 20 frames and number of slices along the long axis of the subjects confine between 8 and 15 and Spacing between slices ranged between 6 and $13 \mathrm{~mm}$. Each image slice consisted of $256 \times 256$ pixels with a pixel spacing of 0.93-1.64 mm. Also the manually segmented images for comparison are available through this literature [4] in some of the slices. In order to check impression of the slice level on segmentation errors, following a standard nomination, three classes of slices have been assigned [26]: apical, mid-ventricular, and basal slice levels [2]. All implementations were done on an Intel (R) core (TM) i7 with 6 GB RAM, using Matlab. The end-diastolic models were applied to frames 1-5, 16-20 and the endsystolic models were applied to frames 6-15.At each slice level, images at ED and ES are selected, for a total of 2,039 images of the LV. The database of short axis cardiac MR images and related manual segmentation that we use was provided by the Department of Diagnostic Imaging of the Hospital for Sick Children in Toronto, Canada [4]. The results are acquired using purposed algorithm and manual ones were compared via calculation of two metric standards as Dice metric and Point to curve error. Dice Metric (DM) [2], measurement of two contours' overlap via following equation:

$$
D M=2 A_{a m}\left(A_{a}+A_{m}\right)^{-1}
$$

Where $A_{a}$ is the area enclosed by the automatic and $A_{m}$ is the area enclosed by manual segmentation and $A_{a m}=A_{a} \cap A_{m}$ is the intersection of both areas. The range of DM is between 0 (the worst amount identifies completely in conformity) and 1 (complete adaption). Point to curve (P2C) error [2], which is the mean perpendicular distance between both contours through following equation:

$$
P 2 C\left(C_{a}, C_{m}\right)=\frac{1}{N_{a}} \sum_{i=1}^{N_{a}} \min d\left(P_{a}^{i}, P_{m}^{j}\right)
$$

where $C_{a}$ identifies automatically obtained contour and $C_{m}$ shows manually segmented contour. For each $C_{a}$ contour point $P_{a}^{i}$, distances to all $C_{m}$ contour points $P_{m}^{i}$ are computed. The minimum distance is retained, and all minima are averaged into $\mathrm{P} 2 \mathrm{C}$ error, over all $N_{a}$ points of contour $C_{a}$. Also $d$ denotes Euclidean distance. A high value of P2C error indicates that contours do not match well.
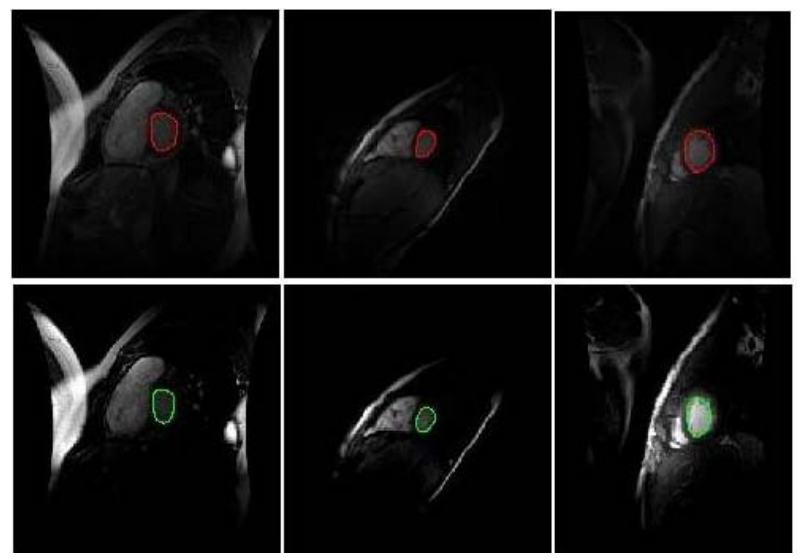

Figure 1: LV Segmentation results on three different patients in ED phase, Red and green contours are obtained with manual and automatic segmentation respectively. Left column: Base, Mid. Column: Mid slice and the right column is related to Apex.
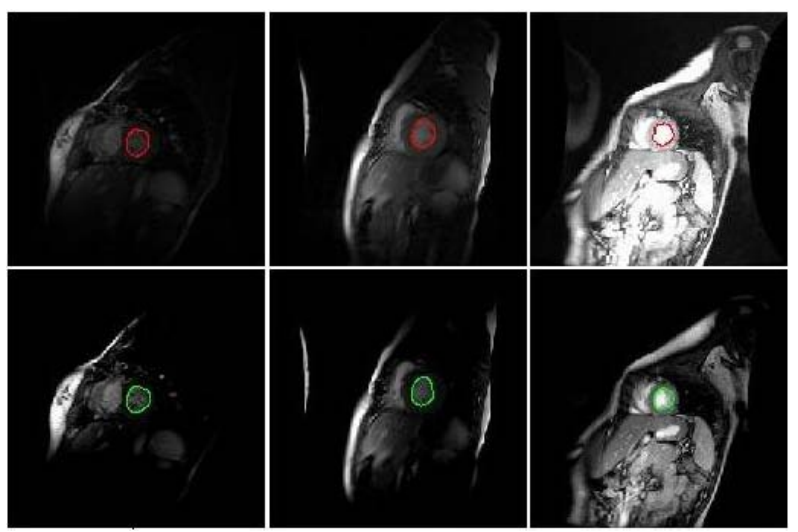

Figure 2: LV Segmentation results on three different patients in ES phase, Red and green contours are obtained with manual and automatic segmentation respectively. Left column: Base, Mid. Column: Mid slice and the right column is related to Apex. 
Fig. 1 shows segmentation results on 3 images in ED phase and Fig. 2 identifies some other segmented results in ES phase. A false segmentation rate was computed according to number of mis-segmentation cases when the manual and automatic contours do not overlap at all. The results of Dice metric and false segmentation rates have been collected in Table 1. Also obtained results for Point to curve error are presented in Table 2 for comparis on with other literatures.

We can also remark that papillary muscles are sometimes considered as part of the myocardium (when they are very close to the wall) by the algorithm, whereas the corresponding manual contouring excludes them from the segmentation, which contributes to increase the P2C errors. [2]

Table 1: Mean Dice Metric \pm standard deviation and obtained false segmentation rates on different slice levels [2]

\begin{tabular}{|c|c|c|c|c|c|}
\hline Authors & $\begin{array}{c}\text { LV } \\
\text { phase }\end{array}$ & $\begin{array}{l}\text { Nb. Of } \\
\text { images }\end{array}$ & $\begin{array}{l}\text { Slice } \\
\text { Level }\end{array}$ & $\overline{\mathrm{DM}}$ & $\begin{array}{l}\text { False } \\
\text { Seg. }\end{array}$ \\
\hline \multirow{6}{*}{$\begin{array}{l}\text { Grosgeorge } \\
\text { et al. }\end{array}$} & \multirow[t]{3}{*}{ ED } & \multirow[t]{3}{*}{569} & Base & $0.82 \pm 0.01$ & 0.07 \\
\hline & & & Mid & $0.75 \pm 0.01$ & 0.02 \\
\hline & & & Apex & $0.67 \pm 0.02$ & 0.14 \\
\hline & \multirow[t]{3}{*}{ ES } & \multirow[t]{3}{*}{469} & Base & $0.70 \pm 0.03$ & 0.16 \\
\hline & & & Mid & $0.58 \pm 0.00$ & 0.11 \\
\hline & & & Apex & $0.46 \pm 0.00$ & 0.35 \\
\hline \multirow{6}{*}{$\begin{array}{l}\text { The } \\
\text { present } \\
\text { study }\end{array}$} & \multirow[t]{3}{*}{ ED } & \multirow[t]{3}{*}{1049} & Base & $0.92 \pm 0.03$ & 0 \\
\hline & & & Mid & $0.86 \pm 0.07$ & 0.009 \\
\hline & & & Apex & $0.86 \pm 0.06$ & 0.0028 \\
\hline & \multirow[t]{3}{*}{ ES } & \multirow[t]{3}{*}{990} & Base & $0.88 \pm 0.03$ & 0.017 \\
\hline & & & Mid & $0.84 \pm 0.05$ & 0.03 \\
\hline & & & Apex & $0.82 \pm 0.06$ & 0.015 \\
\hline
\end{tabular}

Table 2: Mean point to curve errors reported in the literatures and present study for LV segmentation [2]

\begin{tabular}{|l|l|l|l|l|}
\hline Authors & $\begin{array}{l}\text { Nb. } \\
\text { Subj. }\end{array}$ & Phases & $\begin{array}{l}\text { Slice } \\
\text { Level/Nb. }\end{array}$ & P2C(mm) \\
\hline $\begin{array}{l}\text { Lynch et al. } \\
\text { [27] }\end{array}$ & 25 & $\begin{array}{l}\text { Ed, } \\
\text { ES }\end{array}$ & $5-12$ & $0.69 \pm 0.88$ \\
\hline $\begin{array}{l}\text { El Berbari et } \\
\text { al. [1] }\end{array}$ & 13 & ED & 3 & $0.60 \pm 0.30$ \\
\hline Kaus et al.[28] & 121 & ED & $7-10$ & $2.45 \pm 0.75$ \\
\hline & & ES & & $2.84 \pm 1.05$ \\
\hline $\begin{array}{l}\text { Mitchell et } \\
\text { al.[29] }\end{array}$ & 20 & ED & Mid & $1.71 \pm 0.82$ \\
\hline $\begin{array}{l}\text { Mitchel et al. } \\
\text { [30] }\end{array}$ & 56 & ED & $8-14$ & $2.75 \pm 0.86$ \\
\hline $\begin{array}{l}\text { Van Assen et } \\
\text { al.[31] }\end{array}$ & 15 & ED & $10-12$ & $1.97 \pm 0.54$ \\
\hline $\begin{array}{l}\text { Lorenzo et } \\
\text { al.[32] }\end{array}$ & 10 & All & Mid & $2.21 \pm 2.22$ \\
\hline & & ED & & $1.88 \pm 2.00$ \\
\hline $\begin{array}{l}\text { Lotjonen et al. } \\
\text { [33] }\end{array}$ & 25 & ED & $4-5$ & $2.01 \pm 0.31$ \\
\hline $\begin{array}{l}\text { Grosgeorge et } \\
\text { al.[2] }\end{array}$ & $59 a$ & ES & Base & $2.45 \pm 1.57$ \\
\hline & & & Mid & $2.27 \pm 2.02$ \\
\hline & & ED & Base & $2.33 \pm 1.78$ \\
\hline & & Mid & $2.91 \pm 2.25$ \\
\hline $\begin{array}{l}\text { The present } \\
\text { study }\end{array}$ & 18 & ES & Base & $1.54 \pm 0.56$ \\
\hline
\end{tabular}

\begin{tabular}{|l|l|l|l|l|}
\hline & & & Mid & $1.92 \pm 0.70$ \\
\hline & & & Apex & $1.66 \pm 0.83$ \\
\hline & & ED & Base & $1.25 \pm 0.50$ \\
\hline & & & Mid & $1.93 \pm 0.98$ \\
\hline & & & Apex & $1.68 \pm 0.84$ \\
\hline
\end{tabular}

a: It's total number of subjects for both $\mathrm{LV}$ and Right ventricle segmentation.

Considering the DM and the false segmentation rate, better performance obtained in the ED than at ES, and on base slices than mid and apical slices. This can be explained by the fact that at the $\mathrm{ED}$, the ventricles are larger and less sensitive to noise. Results also depend on slice levels: basal and mid ventricular slice levels are simpler to segment than apical levels, where ventricle cavities are really small. Apical slices are more prone to the partial volume effect, a consequence of which is certain fuzziness on the ventricle frontiers.

A solution would be to have an adaptive size of the initial contour, corresponding to the different slice levels. Our algorithm is implemented without any particular optimization and included of balloon force computation, requires less than 2 seconds on a regular PC hardware to segment a single image, which represents less time than the elapsed mentioned time in Grosgeorge et al. [2]

\section{CONCLUSION}

In this paper, a novel region-based active contour method with semi-automatic balloon force computation was applied to segment left ventricle MR images. The algorith m was implemented on a large database consists of 18 subjects with 2039 images. The obtained results were compared with manual segmentation according to two metric standards: Dice metric and Point to curve error. The results are more desirable than previous methods in other literatures. The mean improvement of DM compared to Grosgeorge et al. is $12 \%$ for Base Slice, $18.6 \%$ for Mid and $28.3 \%$ for Apex in ED phase and respectively $25.7 \%, 44.8 \%$ and $78 \%$ for Base,Mid and Apex in ES phase. The mean improvement of P2C compared with Grosgeorge et al. is 46\%, 33.6\% and $52 \%$ in ED phase and 37\%, 15\% and 66.8\% in ES phase respectively for Base, Mid and Apex. The best results were obtained in the ED than ES and on Base slices than Mid and Apex and it's because of LV bigger size in ED phase and Base slice.

On some images, especially at the apex, myocardium borders are very fuzzy and ill-defined, and it is very difficult to rely on the image alone to perform segmentation .So there was mistake segmentation over some of the images which was defined as "False segmentation rate". Obtained results show false segmentation rate less than what Grosgeorge et al. got. The false segmentation rate for ED phase in this study is 0.012 and in results obtained by Grosgeorge et al. is 0.23 , also in ES phase in present study the rate of false segmentation is 0.069 in compared to 0.62 by Grosgeorge et al. [2] Also comparison with other 
literatures according to table 2 identifies imp rovement in mean point to curve error.

\section{REFERENCES}

[1] R. El Berbari, I. Bloch, A. Redheuil, E. Angelini, E. Mousseaux, F. Frouin, A. Herment,"An automated myocardial segmentation in cardiac MRI", IEEE Engineering in Medicine \& Biology Society, Lyon, France, 2007, pp.4508-4511.

[2] D. Grosgeorge, C. Petitjean, J. Caudron, J. Fares, JN. Dacher, "Automatic Cardiac Ventricle Segmentation in MR Images: a Validation Study", International Journal of Computer Assisted Radiology and Surgery, vol. 19, No. 8, 2010, pp. 991-1002.

[3] G. Narayan, K. Nayak, J. Pauly, B. Hu," Singlebreathhold, four-dimensional, quantitative assessment of LV and RV function using triggered, real-time, steady-state free precession MRI in heart failure patients", Journal of Magnetic Resonance Imaging, 2005, pp. 59-66.

[4] A. Andreopoulos, J. K. Tsotsos, "Efficient and Generalizable Statistical Models of Shape and Appearance for Analysis of Card iac MRI", Medical Image Analysis, vol. 12,No. 3, 2008, pp. 335-357.

[5] M. Kass, A. Witkin, D. Terzopoulos, "Snakes:active contour models", International Journal of Computer Vision", vol. 1, 1988, pp. 321-331.

[6] N., Ahuia, N., Xu, R., Bansal,"Object seg mentation using graph cuts based active contours", Computer Vision and Image Understanding ", vol. 107, 2007, pp. 210-224.

[7] V. Caselles, R. Kimmel, G. Sapiro, "Geodesic active contours", in Proceedings of IEEE International Conference on Computer Vision, Boston, MA., 1995, pp. 694-699.

[8] V. Caselles, R. Kimmel, G. Sapiro, "Geodesic active contours", International Journal of Computer Vision, vol. 22, No. 1, 1997, pp. 61-79.

[9] T. Chan, L. Vese, (2001) "Active contours without edges", IEEE Transaction on Image Processing, vol. 10, No. 2, pp. 266-277.

[10] G. P. Zhu, Sh. Q. Zhang, Q. SH. Zeng, Ch. H., Wang, "Boundary-based image segmentation using binary level set method", Optical Engineering, vol. 46, 2007.

[11] K.. Zhang, L. Zhang, H. Song, W. Zhou. (2010) "Active contours with selective local or global segmentation: A new formulation and level set method", Image and Vision Computing, vol. 28, pp. 668-676.

[12] C. M. Li, C. Y. Xu, C. F. Gui, M. D. Fox," level set evolution without re-initialization:a new variational formulation", in IEEE Conference on Computer Vision and Pattern Recognition, San Diego, 2005, pp. 430-436.

[13] N. Paragios, R. Deriche, "Geodesic active contours and level sets for detection and tracking of moving objects", IEEE Transaction on Pattern Analysis and Machine Intelligence, vol. 22, 2000, pp. 1-15.

[14] C. Y. Xu, A. Yezzi, J. L. Prince, "On the relation between parametric and geometric active contours". in processing of 34th asilomar conference on signals and computers, 2000.

[15] A. Vasilevskiy, K. Siddiqi, "Flux-maximizing geometric flows", IEEE transaction on pattern analys is and machine intelligence, vol. 24, 2002, pp. 1565-1578.

[16] J. Lie, M. Lysaker, X. C. Tai, (2006) "A binary level set model and some application to MumfordShah image segmentation", IEEE Transaction on Image Processing, vol. 15, 2006, pp.1171-1181.

[17] D. Mumford, J. Shah, "Optimal approximation by piecewise s mooth function and associated variational problems", Communication on Pure and Applied Mathematics, vol. 42, 1989, pp. 577-685.

[18] C. M. Li, C. Kao, J. Gore, Z. Ding, "Implicit active contours driven by local binary fitting energy", in IEEE Conference on Computer Vis ion and Pattern Recognition, 2007.

[19] A. Tsai, A. Yezzi, A. S. Willsky, "Curve evolution implementation of the Mumford-Shah functional for image segmentation, denoising, interpolation, and magnification", IEEE Transaction on Image Processing, vol. 10, 2001, pp. 1169-1186.

[20] L. A. Vese, T. F. Chan, "A multiphase level set framework for image segmentation using the Mumford-Shah model", International Journal of Computer Vision", vol. 50, 2002, pp. 271-293.

[21] R. Ronfard,"Region-based strategies for active contour models", International Journal of Computer Vision, vol. 46, 2002, pp. 223-247.

[22] N., Parag ios, R., Deriche, "Geodesic active regions and level set methods for supervised texture segmentation", International Journal of Computer Vision, vol. 46, 2002, pp. 223-247.

[23] S. Osher, R. Fedkiw, Level Set Method and Dynamic Implicit Surfaces, Applied Mathematical Sciences, Vol. 153, Springer-Verlag, New York, 2002 .

[24] G. Aubert- P. Kornprobst ,"Mathematical Problems in Image Processing: Partial Differential Equations and the Calculus of Variations", Springer Verlag, Applied Mathematical Sciences, vol. 147, 2001.

[25] C. Li, C. Kao, J. C. Gore, Z. Ding, "Minimization of region-scalable fitting energy for image segmentation", in IEEE Transaction on Image Processing, 2008, pp. 1940-1949.

[26] M.D. Cerqueira, N. J. Weissman, V., Dilsizian, A.K., Jacobs, S., Kaul, W K., Laskey, D. J., Pennell, J.A., Rumberger, T., Ryan, M. S., Verani, "Standardized myocardial segmentation and nomenclature for tomographic imaging of the heart", Circulation, vol. 105, No. 4, 2002, pp.539-542.

[27] M. Lynch, O. Ghita, P. Whelan, "Automatic segmentation of the left ventricle cavity and myocardium in MRI data", Computers in bilogy and medicine, vol. 36, No. 4, 2006, pp. 389-407. 
[28] M. R. Kaus, J. Von Berg, J. Weese, W. Niessen,V. Pekar, "Automated segmentation of the left ventricle in card iac MRI, Medical Image Analysis, vol. 8, No. 3, 2004, pp. 245-254.

[29] S. Mitchell, B. Lelieveldt, R. Van der Geest, J. Bosch, J. Reiber, M. Sonka, "Multistage hybrid active appearance model matching: segmentation of left and right ventricles in cardiac MR images", IEEE Transactions of Medical Imaging, vol. 20, No. 5, 2001, pp. 415-423.

[30] S. Mitchell, J. G. Bosch, B. Lelieveldt, R. J. Van der Geest, J. H. C. Reiber, M. Sonka, "3D active appearance models: seg mentation of cardiac MR and ultrasound images", IEEE Transactions of Medical Imaging, vol. 21, No. 9, 2002, pp. 1167-1178.

[31] H. C. Van Assen, M. H. Danilouchkine, A.F. Frangi, S. Orda, Westen berg, J.J. Reiber, J.H. Lelieveldt, B.P. "SPASM: a 3D-ASM for segmentation of sparse and arbitrarily oriented cardiac MRI data", Medical Image Analysis, vol. 10, 2006, pp. 286-303.

[32] M. Lorenzo-Valdes, G. I. Sanchez-Ortiz, A. G. Elkington, R. H. Mohiaddin, D. Rueckert, "Segmentation of 4D cardiac MR images using a probabilistic atlas and the EM algorithm", Meical Image Analysis, vol. 8, No. 3, 2004, pp. 255-265.

[33] J. Lötjönen, S. Kivistö, J. Koikkalainen, D. Smutek, K. Lauerma, "Statistical shape model of atria, ventricles and epicardium from short- and long-axis MR images", Medical Image Analysis, vol. 8, No. 3, 2004, pp. 371-386.

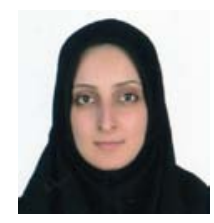

Maryam Aghai Amirkhizi was born in Tabriz, Iran in 1981. She received B.Sc. in Electronic Engineering from Islamic Azad University, Tabriz Branch in 2004 and M.Sc. degree in Mechatronic Engineering from Islamic Azad University, Tabriz Branch in 2013.

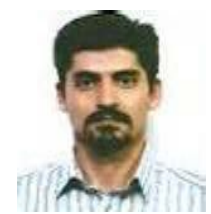

Siyamak Haghipour was born in Urmia, Iran in 1974. He received his bachelor degree from Urmia University, Iran, in 1996, in Electrical Engineering (Major Option in Communication Systems). He got his MSc degree from Iran University of Science \& Technology, Iran, in 1999 and Ph.D. degree from Islamic Azad University, Science and Research Branch, Iran, in 2006 all in BiomedicalBioelectric Engineering. His basic research is about Signal \& Image Processing, Artificial Intelligence, Bioinformatics and Biological Systems Modeling. 